\title{
No-Reference Image Quality Metric Based on Features Evaluation
}

\author{
Omar Alaql, Kambiz Ghazinour, Cheng Chang Lu \\ Department of Computer Science, \\ Kent State University, Kent, Ohio, USA \\ \{oalaql,kghazino,clu\}@kent.edu
}

\begin{abstract}
We develop an efficient general-purpose noreference (NR) image quality assessment (IQA) model that based on a hypothesis that an effective combination of image features can be used to develop NR-IQA approaches having competitive performance with the state-of-the-art. First, we design a collection of features, then evaluate the usefulness of each feature on different kinds of distortions using different features evaluation techniques. Therefore, we came up with a set of optimal features for each learning model in the framework. Our experimental results show that our approach outperforms state- of-the-art blind image quality prediction models.
\end{abstract}

Keywords-component; No Reference Image Quality Assessment (NR-IQA)

\section{INTRODUCTION}

The last decade has witnessed great advances in digital images. Massive numbers of digital images are being captured by mobile digital cameras due to the increasing popularity of mobile imaging devices. These images are subjected to many processing stages during storing, transmitting, or sharing over a network connection. Unfortunately, these processing stages could potentially add visual degradation to original image. These degradations reduce the perceived visual quality which leads to an unsatisfactory experience for human viewers. Therefore, Image Quality Assessment (IQA) has become a topic of high interest and intense research over the last decade. The aim of IQA is to automatically assess image quality in agreement with human judgments. IQA algorithms are divided into the following categories based on the availability of the reference image: Full Reference (FR-IQA), Reduced Reference (RRIQA), and No Reference (NR-IQA), where FR-IQA has full access to the reference image and NR-IQA has no information about the reference image and RR-IQA provides a solution in between.

This paper mainly focuses on the most challenging category of IQA - general-purpose No-Reference IQA (NRIQA), where the goal is to assess the quality of images without information about the reference images and without prior knowledge about the types of distortions in the tested image. We have studied this problem based on a hypothesis that an effective combination of image features can be used to develop NR-IQA approaches having competitive performance with the state-of-the-art.
A two-stages framework, is proposed by Moorthy and Bovik [1], which first classifies the distortion using a classification model then predict the quality value using regression models. In this paper, we improve the regression portion by using different set of features for each distortion instead of using same features for all distortions. We started with features collection that describe different types of distortions in a distorted image. Then, train a model for each distortion type based on these features by trying different regression methods. After that, the best performing regression methods are used to reduce the number of features by investigating different attribute evaluators. Finally, we end up with optimal set of features and a trained model for each distortion type to predict the final quality values. Figure 1 shows the structure of the proposed approach with the number of features that resulted from our evaluation process for each model.

This paper is organized as follows. Section II presents the flowchart of the framework structure. Section III presents the databases that are used in this work. Section IV briefly describes each feature collection and the related work. Section V describes the features evolution techniques. Section VI presents the results of features reduction. Experimental results are presented in Section VII. Finally, the conclusion is described in Section VIII.

\section{THE FRAMEWORK STRUCTURE}

The proposed approach is based on Moorthy and Bovik's framework [1], which has two steps; classification and regression. For classification, the tested image is classified into one of five distortion types in LIVE database. However, it does not conduct a hard decision in classification, but instead it calculates the probability that the image belong to each distortion type. These probabilities are used as weight values to indicate the amount of each distortion in the image. In this work, we use our classification model that is proposed in [2][3]. In the regression stage, the final quality score based on five trained models; one model for each distortion type. Each model predicts a quality value that is multiplied by the associated weight value in classification stage to get quality score for each distortion. Therefore, the final quality score will be the summation of all these scores (Equation 1).

$$
\text { Quality Score }=\sum_{k=1}^{5} w_{i} \cdot q_{i}
$$




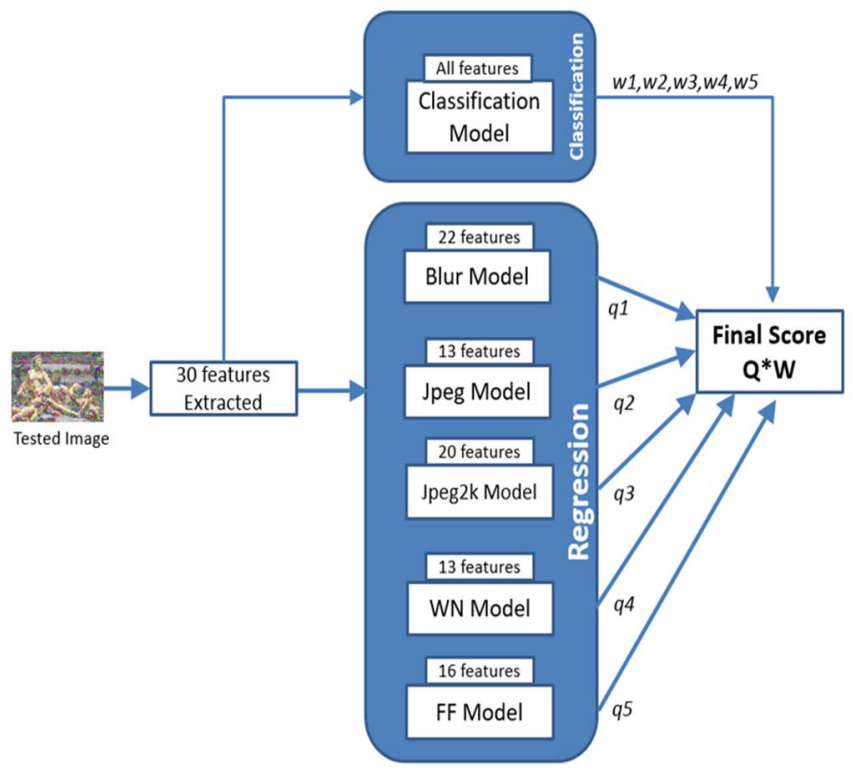

Figure 1: The proposed method structure which shows the number of features for each model.

The features that used in these models are resulted from a validation process that has been done in this research to select the optimal features for each model in the framework. Figure 1 shows the structure of the proposed framework.

\section{DATABASES}

In this research, we have used four image quality databases, which are publically available. These databases are summarized as follows:

(1) LIVE Image Quality Assessment Database [4][5][6]: This database is developed at the Laboratory for Image and Video Engineering (LIVE) at The University of Texas at Austin. It contains 779 distorted images and 29 reference images. There are five different types of distortions in the database, and each type has different amounts of distortions: additive Gaussian white noise (145 distorted), JPEG compression (169 distorted images), JPEG2000 compression (175 distorted images), Gaussian blurring (145 distorted images), and bit errors in JPEG2000 bit stream (145 distorted images).

(2) VCL@FER Image Quality Database [7]: This database is developed by Video Communication Laboratory at the University of Zagreb, Croatia. It contains 552 distorted images and 23 reference images. There are four types of distortion in the database: JPEG compression (138 images), JPEG2000 compression (138 images), white noise (138 images), and Gaussian blur (138 images). The total number of images is 575 .

(3) Tampere Image Database 2013 (TID2013) [8] : The database contains 25 reference images that were used to generate 3000 distorted images. These distorted images contain 24 type of distortions, and each distortion has five levels of distortions.

(4) LIVE Multiply Distorted Image Quality Database [9]: each image in this database contains two type of distortions in the same image. The database has two groups: image storage where images are first blurred and then compressed by a JPEG encoder, and image storage where images are first blurred and then corrupted by white Gaussian noise. Each group contains 225 images.

\section{THE COLLECTION OF FEATURES AND RELATED WORK}

We design a collection of features which describe a set of distortions that the image is subjected to. We focus here on the same set of distortions in LIVE database: JPEG, JPEG2000 (JP2K), white noise (WN), Gaussian Blur (Blur) and Fast fading (FF). 197 features are collected from state of the art. These features are summarized as follows:

\section{A. BIQI:}

In [1] Moorthy and Bovik presented the BIQI algorithm which estimates quality based on a wavelet transform using the Daubechies 9/7 wavelet basis [10]. The transform is performed over three scales and three orientations. The subband coefficient of the transform is parametrized using a generalized Gaussian distribution GGD. There are three parameters in the distribution: mean, variance and shapeparameter. Two parameters only are used: variance and shapeparameter because the mean in the wavelet bases is zero. Therefore, three scales, three orientations, and two parameters generate 18 features for the image $(3 \times 3 \times 2)$. After extracting the features, the tested image is classified by multiclass SVM classifier to one of five classes that represent five different distortions (Jpeg, peg2000, white noise, fast feading error, and blur). This classifier is not only giving an absolute classification, but also giving the amount of each distortion present in the image. To compute the quality score, support vector regression (SVR) is utilized to learn a mapping from the feature space to subjective quality.

\section{B. SSEQ:}

In [11], Liu et al. presented the SSEQ algorithm which estimates quality based on image entropy since the type and 
the amount of the distortion affect the local entropy of images. The features are computed from the spatial entropy and the spectral entropy. The spatial entropy is the probability distribution of the local pixel values, on the other hand, spectral entropy is the probability distribution of the local DCT coefficient values. To extract the features, the mean and the skew of the histogram for both entropies are used to describe the distortion. Moreover, the features are extracted from different scale: low, middle and high. As a result, we have 12 features are obtained using this method ( 2 entropy $\mathrm{x}$ 2 for mean and skew x 3 scales). For the machine learning part, they have used the two stage framework that was proposed in previous algorithm [1]; SVM for classification and SVR for repression.

\section{Curvelet:}

In [12], Liu et al. proposed an algorithm based on curvelet transform [13][14]. The curvelet coefficient is the convolution of the curvelet with an image. Each curvelet has a scale, angle, and location. If a curvelet is aligned on a curve in the image, the curvelet coefficient will be large and vice versa. There are 12 features are extracted using this method. The first four features are extracted by using four parameters in an asymmetric generalized Gaussian distribution model that is used in fitting the histogram of the curvelet coefficient. These parameters are amplitude, mean, and two standard deviations. Two more features are extracted from the orientation energy distribution. The mean kurtosis is one feature, and the second one is the coefficient of variation of the non- cardinal orientation energies which is the standard deviation over the sample mean of the non-cardinal orientation energies. Furthermore, six features are extracted from the scalar energy distribution by calculating energy differences across six scales. The resulted features are used for machine learning stage by using the two steps framework in [1].

\section{BLIINDS-II:}

In [15][16], Saad et al. presented a model that operates entirely in the Discrete Cosine Transform (DCT) domain. The features are extracted in local frequency of the (DCT) domain because the statistics of DCT coefficients affected by changing the type and the amount of the distortion. A new parametric model is proposed to model the extraction of local DCT coefficient in order to use its parameters as resulted features. The total number of features that are used from this method is 24 features.

\section{E. BRISQUE:}

In [17], Mittal et al. proposed the BRISQUE algorithm based on a spatial natural scene statistics model. In this model, the mapping to a different co-ordinate domain is not required, such as wavelet and DCT. Therefore, the computational complexity of extracting these features is very low. The model computes locally normalized luminances, then uses its parameters as a resulted features. The shape and the variance of fitting the generalized Gaussian distribution (GGD) to mean subtracted contrast normalized MSCN coefficients are the first two features. Then, the fitting of asymmetric generalized Gaussian distribution to the pairwise products of neighboring MSCN coefficient along four orientations generates more 16 features. Four features are extracted for each orientation; shape, two variance, and mean. As a result, we have 18 features obtained using this method. These feature are used in a regression module to learn a mapping from feature space to quality scores.

\section{F. DIIVINE:}

In [18], Moorthy and Bovik presented DIIVINE algorithm. The features here are extracted by decomposing the distorted image first using a wavelet decomposition that uses a steerable pyramid over two scales and six orientations $(0,30,60,90,120,150)$. They found that an increased degree of orientation improves the performance.

the subband coefficients across these orientations and scales are then used to extract a set of statistical features. There are in total 88 features used in this method. Then, the quality is estimated by using same two steps framework in [1].

\section{G. Deblurring:}

In [19], Liu et al. designed a framework that used to measure the performance of deblurring algorithms. They made a collection of features, then select an optimal subset of these features based on a validation test. In our research, these optimal features are added to our feature collection. The selected features are: Sparsity priors [20], Gradients of small magnitudes, MetricQ [21], Saturation, Auto-correlation, Cumulative Probability of Blur Detection [22], and Normalized Sparsity Measure [23].

\section{FeAtURES SELECTION TECHNIQUES}

Feature selection is the process of selecting a subset of features in order to enhance generalization. Moreover, feature selection removes redundant and irrelevant features. Since we have large features collection, we have used 17 features selection techniques to generate an optimal subset of features to construct each model. We have done a validation experiment to test the performance of these techniques using LIVE database. The best performing techniques are given below:

\section{A. ClassifierSubsetEval:}

Evaluate subset of features by estimating the merit using classifiers [24] [25].

\section{B. CfsSubsetEval:}

This technique select a subset of features which have high correlation with the class and have low correlation between each other [24]. 


\section{WrapperSubsetEval:}

This method generates subsets of features by using a learning scheme. Then, each subset is validated using cross validation to select the best set which has the highest accuracy rate [26].

\section{ConsistencySubsetEval:}

It is based on the level of consistency in the class values. It uses Random or Exhaustive search method to look for the smallest subset with consistency equal to consistency of the full set of attributes since the consistency of any subset can not be lower than consistency of the full set of attributes [27].

\section{OPTIMAL FEATURES SELECTION}

Before evaluating the features, we have investigated the performance of different regression methods to come up with the best performing one using our feature collection. Table 1 shows the best performing regression methods. The LIVE database is used here with 10 folds cross validation. The best performing regression method is Linear Regression. This regression got Correlation coefficient of 0.8977 .

Table 1: The best performing Regression Methods using LIVE database

\begin{tabular}{|c|c|c|c|c|c|c|}
\hline \multirow{2}{*}{$\begin{array}{c}\text { Regression } \\
\text { Methods }\end{array}$} & \multicolumn{6}{|c|}{ Correlation coefficient } \\
\cline { 2 - 7 } & Blur & FF & Jpeg2K & Jpeg & WN & ALL \\
\hline $\begin{array}{c}\text { epsilon } \\
\text { SVR, linear }\end{array}$ & 0.9409 & 0.8447 & 0.8883 & 0.8530 & 0.9625 & 0.8421 \\
\hline $\begin{array}{c}\text { nu SVR, } \\
\text { linear }\end{array}$ & 0.9374 & 0.8398 & 0.8878 & 0.8601 & 0.9719 & 0.8418 \\
\hline $\begin{array}{c}\text { Linear } \\
\text { Regression }\end{array}$ & 0.9587 & 0.8951 & 0.9321 & 0.8390 & 0.9895 & 0.8977 \\
\hline
\end{tabular}

Table 2: The resulted features from different evaluators using LIVE database

\begin{tabular}{|c|c|c|c|c|c|}
\hline \multirow{2}{*}{ Evaluation method } & \multicolumn{5}{|c|}{ Correlation coefficient } \\
\cline { 2 - 6 } & Blur & FF & Jpeg2K & Jpeg & WN \\
\hline CfsSubSetEval & 0.935 & 0.8478 & 0.8964 & 0.8603 & 0.9901 \\
\hline $\begin{array}{c}\text { ClassifierSubsetEval, } \\
\text { Linear Reqression }\end{array}$ & 0.9599 & 0.9169 & 0.9347 & 0.8529 & 0.9924 \\
\hline $\begin{array}{c}\text { ClassifierSubsetEval, } \\
\text { SVR }\end{array}$ & 0.9446 & 0.8692 & 0.9153 & 0.8438 & 0.9906 \\
\hline $\begin{array}{c}\text { WrapperSubsetEval, } \\
\text { Linear Regression }\end{array}$ & 0.9652 & 0.8941 & 0.9383 & 0.8665 & 0.9924 \\
\hline
\end{tabular}

For each distortion type, we have investigated different attributes evaluators to select the optimal subset of features for each one in order to reduce the overfitting. These evaluators select the optimal subset of features. Table 2 shows a number of features sets that are resulted from different evaluation methods using LIVE database. The features that are obtained from classifier subset evaluator with Linear Regression as a base classifier is best performing set of features. The set has only 13 features for white noise, 13 features for Jpeg, 20 features for Jpeg2K, 16 features for
Fast Fading, and 22 features for blur. Table 3 shows each model's features in more details. By comparing the correlation coefficient in Table 1 and Table 2, we can see the results are improved even though we have reduced features.

Table 3: Description of each resulted feature in the classification model and in which regression model is used

\begin{tabular}{|c|c|c|}
\hline $\begin{array}{l}\text { Features } \\
\text { Set }\end{array}$ & $\begin{array}{c}\text { Features Description } \\
\text { (fn: } n \text { is the feature number in the reference paper) }\end{array}$ & $\begin{array}{c}\text { Regression } \\
\text { Model }\end{array}$ \\
\hline \multirow{3}{*}{ BIQI } & $f 7$ : the shape parameter on the horizontal orientation. & Blur \\
\hline & f12: the mean parameter on the diagonal orientation. & FF J2K JPEG \\
\hline & f15: the variance parameter on the diagonal orientation & Blur FF JPEG \\
\hline \multirow{2}{*}{ SSEQ } & F3: the mean of spatial entropy value of the third scale, & $\begin{array}{l}\text { Blur J2K } \\
\text { JPEG WN }\end{array}$ \\
\hline & f11: the skew of spectral entropy value of the second scale. & Blur J2K \\
\hline Curvelet & $\begin{array}{l}f 10 \text { :it is the feature that describe the scalar energy } \\
\text { distribution on the forth dimension, }\end{array}$ & $F F J 2 K W N$ \\
\hline \multirow{6}{*}{ Brisque } & $\begin{array}{l}f 1 \text { : shape parameter which is computed by fitting } \\
\text { generalized Gaussian distribution (GGD) to the mean } \\
\text { subtracted contrast normalized (MSCN) coefficient for the } \\
\text { first scale. }\end{array}$ & FF JPEG \\
\hline & $\begin{array}{l}f 5 \text { : left variance paremeter which is computed by fitting } \\
\text { asymmetric generalized Gaussian distribution (AGGD) to } \\
\text { horizontal pairwise products for the first scale. }\end{array}$ & $\begin{array}{c}\text { Blur FF J2K } \\
\qquad W N\end{array}$ \\
\hline & $\begin{array}{l}f 7 \text { :shape parameter which is computed by fitting AGGD to } \\
\text { vertical pairwise products for the first scale. }\end{array}$ & $\begin{array}{l}\text { Blur FF J2K } \\
\text { JPEG WN }\end{array}$ \\
\hline & $\begin{array}{l}f 10 \text { : right variance parameter which is computed by fitting } \\
\text { AGGD to vertical pairwise products for the first scale. }\end{array}$ & Blur J2K WN \\
\hline & $\begin{array}{l}\text { f15: shape parameter which is computed by fitting AGGD } \\
\text { to the main diagonal pairwise products for the first scale. }\end{array}$ & $\begin{array}{l}\text { Blur FF J2K } \\
\text { JPEG WN }\end{array}$ \\
\hline & $\begin{array}{l}f 23 \text { : left variance paremeter which is computed by fitting } \\
\text { AGGD to horizontal pairwise products for the second } \\
\text { scale. }\end{array}$ & Blur FF \\
\hline \multirow{4}{*}{ Divine } & $\begin{array}{l}f 8 \text { : variance of the } 8^{\text {th }} \text { subband coefficient which is } \\
\text { computed by fitting a generalized Gaussian to the subband } \\
\text { coefficient. }\end{array}$ & Blur J2K WN \\
\hline & $\begin{array}{l}f 13, f 14, f 15, f 21: \text { shape paremeter of the } 1^{\text {st, }} 2^{\text {nd }}, 3^{\text {rd }} \text {, and } \\
9^{\text {th }} \text {, respectively , subband coefficient which is computed } \\
\text { by fitting a generalized Gaussian to the subband } \\
\text { coefficient. }\end{array}$ & $\begin{array}{c}\operatorname{Blur}(21) \\
F F(13) \\
J 2 K(14,15) \\
J P E G(21) \\
W N(13,14)\end{array}$ \\
\hline & $\begin{array}{l}f 50, f 63, f 69 \text { : these are computed from spatial correlation } \\
\text { across different subbands by fitting a polynomial to a } \\
\text { correlation function. }\end{array}$ & $\begin{array}{l}\text { Blur }(63,69) \\
F F(50) \\
J 2 K(63) \\
J P E G(50)\end{array}$ \\
\hline & $\begin{array}{l}f 82 \text { : it is across orientation feature which is extracted by } \\
\text { computing windowed structural correlation between all } \\
\text { possible pairs of subbands at the coarsest scale. }\end{array}$ & Blur FF \\
\hline \multirow{7}{*}{ Bliinds2 } & $\begin{array}{l}\text { f1: Coefficient of variation for 100th percentile on the first } \\
\text { scale. }\end{array}$ & $\begin{array}{l}F F J 2 K J P E G \\
W N\end{array}$ \\
\hline & $\begin{array}{l}\text { f2: Coefficient of variation for 10th percentile on the first } \\
\text { scale. }\end{array}$ & $\begin{array}{c}\text { Blur FF J2K } \\
\text { JPEG WN }\end{array}$ \\
\hline & f3: Shape parameter for 100 th percentile on the first scale. & Blur J2K \\
\hline & $\begin{array}{l}f 5: \text { Energy subband ratio measure for 100th percentile on } \\
\text { the first scale. }\end{array}$ & $\begin{array}{c}\text { Blur J2K } \\
\text { JPEG WN }\end{array}$ \\
\hline & $\begin{array}{l}f 8: \text { orientation parameter for } 10 \text { th percentile on the first } \\
\text { scale. }\end{array}$ & $\begin{array}{l}\text { Blur JPEG } \\
\quad F F\end{array}$ \\
\hline & $\begin{array}{l}\text { f19: Coefficient of variation for 100th percentile on the } \\
\text { third scale. }\end{array}$ & $\begin{array}{l}\text { Blur JPEG } \\
\quad J 2 K\end{array}$ \\
\hline & $\begin{array}{l}f 20 \text { : Coefficient of variation for } 10 \text { th percentile on the third } \\
\text { scale. }\end{array}$ & $\begin{array}{c}\text { Blur FF J2K } \\
W N\end{array}$ \\
\hline \multirow{2}{*}{ Deblurring } & f4: Auto-correlation. & Blur J2K \\
\hline & f6: Cumulative Probability of Blur Detection. & Blur FF J2K \\
\hline
\end{tabular}




\section{EXPERIMENTS AND RESULTS}

In this section, we empirically evaluate our model and present an evaluation of generalizing across many distortion types. We perform evaluation on four databases; LIVE ,TID20113, VCL@FER, and LIVE-MultiDistortions datasets. The total of images in these databases is 4804 .

The first experiment is conducted to validate how the objective assessment of our model corresponds to human evaluation. LIVE database is used in this experiment to train and test the proposed model. We use cross validation method with 10-folds and multiple runs. Table 4 shows the resulted Spearman correlation between the predicted scores values and the subjective scores.

Table 2 Spearman correlation using 10-folds Cross validation performed through LIVE database

\begin{tabular}{|c|c|c|c|c|c|}
\hline Blur & Jpeg & Jpeg2K & FF & WN & All \\
\hline 0.9103 & 0.8269 & 0.9273 & 0.8645 & 0.9840 & $\mathbf{0 . 8 8 9 3}$ \\
\hline
\end{tabular}

The second experiment is conducted to verify the extensibility and generalization of the proposed method. We train metrics on one database and test them on the other databases. Four methods are employed to compare: BIQI[1], BRISQUE [17], SSEQ [11], BLIINDS-II [15].

To make a fair comparison, we train our method on the LIVE database as well and test all the methods on TID20113, and VCL@FER databases to prove whether the proposed method is applicable for various images and distortions without extra training. First, we test the trained methods on the VCL@FER database where all the types of distortion are existing in the training database. It is observed that most of the test methods show relatively good performance across the database as shown in Table 5. In average, our method outperforms most of them, and close to the best one (SSEQ). It outperforms the others for two types of distortions, while SSEQ outperforms for only one distortion. This demonstrates that the proposed method is robust against different image databases.

Table 3: Spearman correlation coefficient on VCL@FER database

\begin{tabular}{|c|c|c|c|c|c|}
\hline Noise Type & $\begin{array}{c}\text { Our } \\
\text { metric }\end{array}$ & BIQI & Brisque & SSEQ & Bliinds2 \\
\hline blur & $\mathbf{0 . 8 9 0 8}$ & 0.6396 & 0.8530 & 0.8822 & 0.8530 \\
\hline JPEG & $\mathbf{0 . 8 2 1 7}$ & 0.5733 & 0.7650 & 0.8104 & 0.7650 \\
\hline JPEG2K & 0.7202 & 0.5833 & 0.7333 & $\mathbf{0 . 8 5 9 7}$ & 0.7333 \\
\hline WN & 0.8116 & 0.7027 & $\mathbf{0 . 8 9 3 4}$ & 0.7494 & 0.8934 \\
\hline ALL & $\mathbf{0 . 8 1 1 1}$ & $\mathbf{0 . 6 2 4 7}$ & $\mathbf{0 . 7 8 3 7}$ & $\mathbf{0 . 8 2 5 4}$ & $\mathbf{0 . 7 8 3 7}$ \\
\hline
\end{tabular}

Second, we test the trained models on TID2013 database where many distortion types do not appear in the training dataset. In many cases, the majority fails. This is because the training data set does not contain these kinds of distortion. However, the proposed metric is the best performing method. It outperforms the others for eight distortion types, while the second best performing one outperforms for only five distortion types as shown in Figure 2 and Table 6 . Furthermore, the proposed mothed gets the best correlation score in average for all images in the database. This result proves that our metric has better generalization capability, because the features evaluator that is used here to get the features in our model increases the generalization capability and decreases the overfitting.

The third experiment is conducted to verify the capability of the proposed method to assess images contain mixtures of multiple distortions. All the tested metrics are trained on LIVE database and tested on LIVE Multiply Distorted Image Quality Database which contains two groups of images and each group contain a mixture of two type of distortions. Table 7 shows the Spearman correlation between the predicted scores and the human judgment for the five tested metrics. The obtained results show clearly that our metric gives better results and yields a significant improvement of the correlation coefficients.

Table 4: Spearman correlation coefficient on TID2013 database

\begin{tabular}{|c|c|c|c|c|c|}
\hline Noise Type & $\begin{array}{l}\text { Our } \\
\text { metric }\end{array}$ & BIQI & Brisque & SSEQ & Bliinds2 \\
\hline $\begin{array}{c}\text { 1-Additive Gaussian } \\
\text { noise }\end{array}$ & 0.8141 & 0.7842 & 0.8520 & 0.8082 & 0.7233 \\
\hline $\begin{array}{l}\text { 2-Additive noise in } \\
\text { color components }\end{array}$ & 0.6968 & 0.5668 & 0.7089 & 0.6821 & 0.6497 \\
\hline $\begin{array}{c}\text { 3-Spatially correlated } \\
\text { noise }\end{array}$ & 0.5912 & 0.3755 & -0.4916 & 0.6311 & 0.7674 \\
\hline 4-Masked noise & 0.8011 & 0.6312 & 0.6012 & 0.6674 & 0.4157 \\
\hline 5-High frequency noise & 0.8376 & 0.8778 & 0.6066 & 0.8418 & 0.7484 \\
\hline 6-Impulse noise & 0.7405 & 0.6890 & 0.6391 & 0.8456 & 0.6556 \\
\hline 7-Quantization noise & 0.7672 & 0.2962 & 0.7053 & 0.4570 & 0.7027 \\
\hline 8-Gaussian blur & 0.8509 & 0.8094 & 0.8469 & 0.8457 & 0.8624 \\
\hline 9-Image denoising & 0.7418 & 0.4121 & 0.6052 & 0.7528 & 0.6802 \\
\hline 10-JPEG & 0.8321 & 0.8826 & 0.8649 & 0.7943 & 0.8494 \\
\hline 11-JPEG2000 & 0.9016 & 0.8223 & 0.8555 & 0.8720 & 0.8622 \\
\hline 12-JPEG errors & 0.4383 & 0.3657 & 0.4254 & 0.4617 & 0.2241 \\
\hline 13-JPEG2000 errors & 0.5454 & 0.3717 & 0.4213 & 0.6180 & 0.6898 \\
\hline $\begin{array}{c}\text { 14-Non eccentricity } \\
\text { pattern noise }\end{array}$ & 0.1050 & 0.0287 & 0.2023 & 0.0582 & 0.1585 \\
\hline $\begin{array}{l}\text { 15-Local block-wise } \\
\text { distortions of different } \\
\text { intensity }\end{array}$ & 0.2990 & -0.147 & 0.2656 & 0.0607 & 0.2529 \\
\hline $\begin{array}{l}\text { 16-Mean shift (intensity } \\
\text { shift) }\end{array}$ & 0.2214 & 0.0625 & 0.2253 & 0.2199 & 0.2290 \\
\hline 17-Contrast change & 0.0381 & 0.4802 & 0.0520 & 0.3037 & -0.0593 \\
\hline $\begin{array}{l}\text { 18-Change of color } \\
\text { saturation }\end{array}$ & 0.1158 & -0.140 & 0.2024 & 0.1680 & 0.1132 \\
\hline $\begin{array}{l}\text { 19-Multiplicative } \\
\text { Gaussian noise }\end{array}$ & 0.7569 & 0.6287 & 0.6412 & 0.7377 & 0.6682 \\
\hline 20-Comfort noise & 0.3498 & 0.2601 & 0.0884 & 0.0563 & 0.0995 \\
\hline $\begin{array}{c}\text { 21-Lossy compression } \\
\text { of noisy images }\end{array}$ & 0.8039 & 0.5264 & 0.6996 & 0.6269 & 0.7418 \\
\hline $\begin{array}{c}\text { 22-Image color } \\
\text { quantization with dither }\end{array}$ & 0.6082 & 0.6812 & 0.6538 & 0.5520 & 0.7245 \\
\hline $\begin{array}{c}\text { 23-Chromatic } \\
\text { aberrations }\end{array}$ & 0.8077 & 0.7170 & 0.7761 & 0.8171 & 0.7173 \\
\hline $\begin{array}{l}\text { 24-Sparse sampling and } \\
\text { reconstruction }\end{array}$ & 0.8149 & 0.7955 & 0.8197 & 0.9086 & 0.8532 \\
\hline ALL & 0.6001 & 0.4907 & 0.5111 & 0.5745 & 0.5554 \\
\hline
\end{tabular}




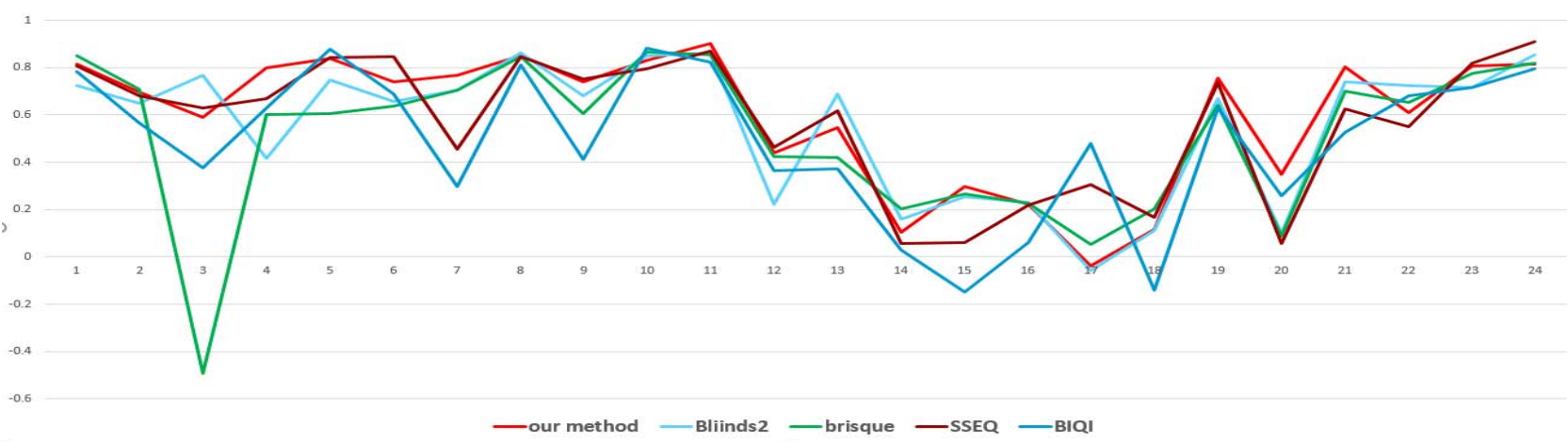

Figure 2 Obtained Spearman correlation coefficient between the predicted values and the subjective values considering TID2013 database as test set. The type of degradations is described in Table 13

Table 5 Spearman correlation coefficient on LIVE Multiply Distorted Image Quality Database

\begin{tabular}{|c|c|c|c|c|c|}
\hline Noise Type & $\begin{array}{c}\text { Our } \\
\text { metric }\end{array}$ & BIQI & Brisque & SSEQ & Bliinds2 \\
\hline Blur Jpeg & 0.8443 & 0.6542 & 0.8453 & 0.2389 & 0.6654 \\
\hline Blur Noise & 0.4837 & 0.4902 & 0.2868 & 0.4367 & 0.0152 \\
\hline ALL & $\mathbf{0 . 6 6 4 0}$ & $\mathbf{0 . 5 7 2 2}$ & $\mathbf{0 . 5 6 6 1}$ & $\mathbf{0 . 3 3 7 8}$ & $\mathbf{0 . 3 4 0 3}$ \\
\hline
\end{tabular}

\section{CONCLUSION}

A blind image quality assessment approach is proposed here using sets of optimal features that are resulted from our features evaluation process. The proposed metric is based on BIQI framework [1]. But instead of using same features in all regression models, we use an optimal set of feature for each distortion model. Different evaluation techniques are used to evaluate the usefulness of each feature. Moreover, we have investigated different classification and regression methods to be used in the learning models. The obtained results show that our metric gives better results and yields a significant improvement of the correlation coefficients with human judgments.

\section{REFERENCES}

[1] A. K. Moorthy and A. C. Bovik, "A two-step framework for constructing blind image quality indices," IEEE Signal Processing Letters, vol. 17, no. 5, pp. 513-516, 2010.

[2] O. Alaql, K. Ghazinour, and C. C. Lu, "Classification of Image Distortions based on Features Evaluation," in IEEE International Symposium on Multimedia (ISM), 2016.

[3] O. Alaql, K. Ghazinour, and C. C. Lu, "Classification Of Image Distortions For Image Quality Assessment," International Conference on Computational Science and Computational Intelligence (CSCI), 2016.

[4] H. Sheikh, Z. Wang, L. Cormack, and A. Bovik, "LIVE image quality assessment database release 2," 2005.

[5] H. Sheikh, M. Sabir, and A. Bovik, "A statistical evaluation of recent full reference image quality assessment algorithms," Image Processing, IEEE ..., 2006.
[6] Z. Wang, A. C. Bovik, H. R. Sheikh, and E. P. Simoncelli, "Image quality assessment: From error visibility to structural similarity," IEEE Transactions on Image Processing, vol. 13, no. 4, pp. 600-612, 2004.

[7] A. Zarić, N. Tatalović, and N. Brajković, "Vcl@, fer image quality assessment database," ... časopis za automatiku ..., 2012.

[8] N. Ponomarenko, O. Ieremeiev, and V. Lukin, "Color image database TID2013: Peculiarities and preliminary results," (EUVIP), 2013 4th ..., 2013.

[9] D. Jayaraman, A. Mittal, and A. Moorthy, "Objective quality assessment of multiply distorted images," Conference Record of ..., 2012.

[10] I. Daubechies, "Ten Lectures on Wavelets, Society for Industrial and Applied Mathematics, 1992.20 I. Daubechies, Ten Lectures on Wavelets," Society for Industrial and Applied Mathematics, 1992.

[11] L. Liu, B. Liu, H. Huang, and A. C. Bovik, "No-reference image quality assessment based on spatial and spectral entropies," Signal Processing: Image Communication, vol. 29, no. 8, pp. 856-863, Sep. 2014.

[12] L. Liu, H. Dong, H. Huang, and A. C. Bovik, "Noreference image quality assessment in curvelet domain," Signal Processing: Image Communication, vol. 29, no. 4, pp. 494-505, 2014.

[13] E. Candes, L. Demanet, D. Donoho, and L. Ying, "Fast discrete curvelet transforms," Multiscale Modeling \& Simulation, 2006.

[14] E. Candès and D. Donoho, "New tight frames of curvelets and optimal representations of objects with piecewise C2 singularities," Communications on pure and applied ..., 2004.

[15] M. A. Saad, A. C. Bovik, and C. Charrier, "Blind image quality assessment: a natural scene statistics approach in the DCT domain.," IEEE transactions on image processing : a publication of the IEEE Signal Processing Society, vol. 21, no. 8, pp. 3339-52, Aug. 2012.

[16] M. Saad, A. Bovik, and C. Charrier, "DCT statistics modelbased blind image quality assessment," Image Processing (ICIP), ..., 2011.

[17] A. Mittal, A. K. Moorthy, and A. C. Bovik, "No-reference image quality assessment in the spatial domain.," IEEE transactions on image processing : a publication of the IEEE Signal Processing Society, vol. 21, no. 12, pp. 4695708, Dec. 2012. 
[18] A. Moorthy and A. Bovik, "Blind image quality assessment: From natural scene statistics to perceptual quality," Image Processing, IEEE Transactions ..., 2011.

[19] Y. Liu, J. Wang, and S. Cho, "A No-Reference Metric for Evaluating the Quality of Motion Deblurring," $A C M$ Transactions on Graphics, vol. 32, no. 6, pp. 1-12, 2013.

[20] A. Levin, R. Fergus, F. Durand, and W. Freeman, "Image and depth from a conventional camera with a coded aperture," ACM Transactions on ..., 2007.

[21] X. Zhu and P. Milanfar, "A no-reference sharpness metric sensitive to blur and noise," Quality of Multimedia Experience, 2009. ..., 2009.

[22] N. Narvekar and L. Karam, "A no-reference image blur metric based on the cumulative probability of blur detection (CPBD)," Image Processing, IEEE Transactions
..., 2011.

[23] D. Krishnan, T. Tay, and R. Fergus, "Blind deconvolution using a normalized sparsity measure," ... Recognition (CVPR), 2011 IEEE ..., 2011.

[24] M. Hall, "Correlation-based feature selection for machine learning," 1999.

[25] I. Witten and E. Frank, Data Mining: Practical machine learning tools and techniques. 2005.

[26] R. Kohavi and G. John, "Wrappers for feature subset selection," Artificial intelligence, 1997.

[27] H. Liu and R. Setiono, "A probabilistic approach to feature selection-a filter solution," ICML, 1996. 